International Journal of Modern Physics B Vol. 30, No. 15 (2016) 1541003 (10 pages)

(C) World Scientific Publishing Company

DOI: $10.1142 / \mathrm{S} 0217979215410039$

\title{
Predator population depending on lemming cycles
}

\author{
Ekaterina I. Anashkina* \\ Faculty of Physics, Lomonosov Moscow State University, \\ Leninskie Gory, 119992 Moscow, Russia \\ Group of Interdisciplinary Theoretical Physics, \\ Università di Palermo and CNISM, Unità di Palermo, \\ Viale delle Scienze, Edificio 18, 90128 Palermo, Italy \\ anashkina@physics.msu.ru \\ Olga A. Chichigina \\ Faculty of Physics and International Laser Center, \\ Lomonosov Moscow State University, Leninskie Gory, 119992 Moscow, Russia \\ chichigina@ilc.edu.ru \\ Davide Valenti \\ Group of Interdisciplinary Theoretical Physics, \\ Università di Palermo and CNISM, Unità di Palermo, \\ Viale delle Scienze, Edificio 18, 90128 Palermo, Italy \\ Istituto Nazionale di Fisica Nucleare, Sezione di Catania, \\ Via S. Sofia 64, I-95123 Catania, Italy \\ davide.valenti@unipa.it \\ Aleksey V. Kargovsky \\ Faculty of Physics and International Laser Center, \\ Lomonosov Moscow State University, Leninskie Gory, 119992 Moscow, Russia \\ kargovsky@yumr.phys.msu.ru \\ Bernardo Spagnolo \\ Group of Interdisciplinary Theoretical Physics, \\ Università di Palermo and CNISM, Unità di Palermo, \\ Viale delle Scienze, Edificio 18, 90128 Palermo, Italy \\ Radiophysics Department, Lobachevsky State University, 23 Gagarin Avenue, \\ 603950 Nizhniy Novgorod, Russia \\ Istituto Nazionale di Fisica Nucleare, Sezione di Catania, \\ Via S. Sofia 64, I-95123 Catania, Italy \\ bernardo.spagnolo@unipa.it
}

*Corresponding author. 


\begin{abstract}
In this paper, a Langevin equation for predator population with multiplicative correlated noise is analyzed. The noise source, which is a nonnegative random pulse noise with regulated periodicity, corresponds to the prey population cycling. The increase of periodicity of noise affects the average predator density at the stationary state.
\end{abstract}

Keywords: Noise in biological systems; dead-time-distorted Poisson process; Langevin equation; population dynamics.

PACS numbers: 05.40.-a, 02.50.Ey, 87.23.kg

\title{
1. Introduction
}

In the last few decades, stochastic differential equations are being widely used in the description of population dynamics. ${ }^{1,2}$ The white noise as a stochastic component is well-investigated, ${ }^{3,4}$ but to model real processes using noises with different statistical properties can be more appropriate.

The predator-prey model is a standard way to describe the dynamics in species population. ${ }^{5}$ This approach works well when we analyze closed populations of predators and prey, but the analytical description becomes quite complex, especially if we include parameters concerning the large fluctuations of prey. Moreover, correct parameters of the model can be found only by experimental results, which provides few information when analysis is performed over short time intervals.

In this work, we suggest a way to describe the time evolution of predator population, whose diet is mainly but not totally based on a species whose number is characterized by quasi-periodic changes in time. More specifically, we describe the population of Arctic fox, whose main prey are lemmings. ${ }^{6}$ They are small rodents basically dwelling in Arctic areas. Lemmings are known for the wide fluctuations in the number of individuals. In particular, the time interval between two successive abundance peaks ranges from three to five years. The causes of population fluctuations are unclear, although some combination of predation, food quantity and quality, and climatic conditions is probably involved. In our model, we neglect the influence of Arctic fox abundance on lemming population, since many other predators also hunt lemmings.

Lemming number can be described from the mathematical point of view as a pulse sequence. ${ }^{7}$ Pulses occur only at years of anomalous increase in population. The periodicity of the process can be regulated from quasi-periodic process to random pulse process. ${ }^{8}$ Specifically, experimental data of lemming populations shows fairly high periodicity with cycles,${ }^{9}$ absence of regularity with random pulses, ${ }^{10,11}$ and something intermediate between these extreme cases (see Fig. 2 of Ref. 8). ${ }^{12,13}$

The main purpose of this work is to describe how the mean population density of predators at the saturation level depends on the periodicity of lemming cycles. 
To describe the changes in predator population we use a Langevin equation with multiplicative noise.

\section{Dynamics of the Predator Population}

In population dynamics, random fluctuations are always modeled as a multiplicative noise source, because the dynamics of the population density is driven by statedependent fluctuation amplitude, ${ }^{4,14-27}$ which allows to reproduce experimental data. This means that, the effects of fluctuations have to be proportional to the activity density, which is the predator density in our system.

Sometimes fluctuations are considered as an additive noise source. ${ }^{28,29}$ But in these cases, some constraints are included in the dynamical model in order to avoid the negative initial random fluctuations or negative large fluctuations during the time evolution of the population.

In our study, we describe the predator population density as a stochastic process $N(t)$. We assume that the effect of fluctuations in the prey density scales as a power of the predator population density, which is less than 1 . We consider the case of the square root of $N(t)$, due to the fact that lemmings are not the only food for Arctic foxes. To describe the predator population we suggest the following form of Langevin equation:

$$
\dot{N}(t)=-b N^{\beta}(t)+\sqrt{N(t)} \xi(t),
$$

which we interpret in the Stratonovich sense. ${ }^{5}$ Here, $N(t)$ is the predator population density, $\beta>1 / 2$, and $\xi(t)$ is the nonnegative pulse noise, which mimics the random variations in the density of lemming population. ${ }^{30}$ The first term on the right-hand side of Eq. (1) determines all processes responsible for the change of population density, except the influence of changes in lemming population. The main of these processes is intraspecific competition, therefore the first term is negative and the higher is the number of individuals, the faster the decrease of predators occurs.

We use noise $\xi(t)$, which is named dead-time-distorted Poisson (DTDP) process and represents an example of renewal pulse process. In particular, this noise is characterized by the presence, after each pulse, of a delay during which the next anomalous increase of population is forbidden, for example, because of the lack of resources such as food or favorable environmental conditions. After this delay, there is some constant probability that a new peak occurs. As a result, cycles with random period take place. Detailed description of the properties of this process is given in Sec. 2.2. Figure 1 shows two sets of experimental data ${ }^{30}$ for the lemming population and an approximation of these data by a DTDP process.

We recall that the dynamics of a system governed by a stochastic differential equation with correlated noise ${ }^{31}$ can be described in stationary state by a source of white noise, provided that corresponding values of the parameters are used..$^{32}$ 


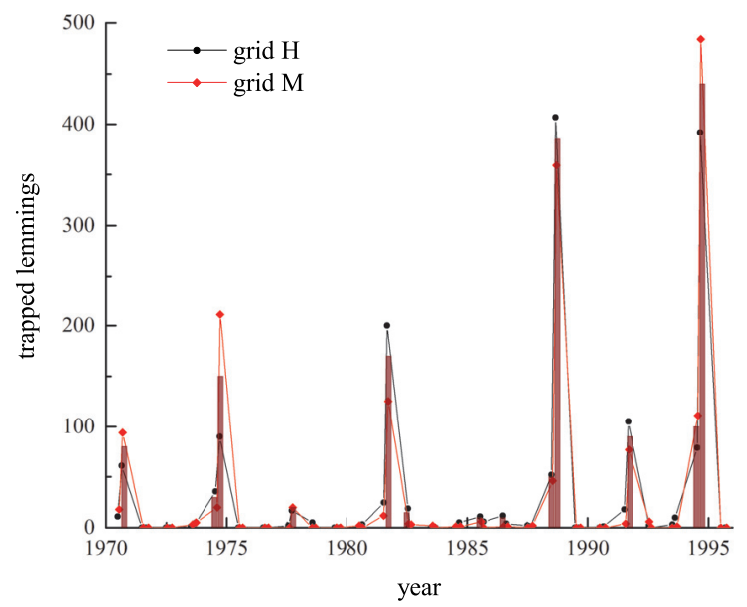

Fig. 1. (Color online) Two different experimental datasets (grid $\mathrm{H}$ and grid $\mathrm{M}$ ) for the lemming population (see Ref. 30) and as an approximated reproduction (vertical bars) of these data by a dead-time-distorted Poisson process.

\subsection{Analytical consideration}

Let us rewrite Eq. (1), extracting the nonzero mean $a=\langle\xi(t)\rangle$ from the noise term. Then, we get

$$
\dot{N}(t)=a \sqrt{N(t)}-b N^{\beta}(t)+\sqrt{N(t)} \eta(t)
$$

Here, we assume that $\eta(t)$ is a stationary Gaussian white noise with $\langle\eta(t)\rangle=0$ and $\langle\eta(t) \eta(t+\tau)\rangle=2 D \delta(\tau)$.

The corresponding Fokker-Planck equation for the probability density function (PDF) $w(N, t)$ has the following form:

$$
\frac{\partial w(N, t)}{\partial t}=-\frac{\partial}{\partial N}\left[\left(a \sqrt{N}-b N^{\beta}+\frac{D}{2}\right) w(N, t)\right]+D \frac{\partial^{2}}{\partial N^{2}}[N w(N, t)]
$$

with reflecting boundary at $N=0$ (the probability flux through the boundary is zero).

It is easy to obtain the stationary solution of Eq. (3) as

$$
w(N)=\frac{\mathcal{N}}{\sqrt{N}} \exp \left(\frac{2 a}{D} \sqrt{N}-\frac{b}{\beta D} N^{\beta}\right)
$$

where $\mathcal{N}$ is the normalization constant. Using Ref. 33, we obtain the probability density function for $\beta=1$

$$
w(N)=\sqrt{\frac{b}{\pi D N}} \frac{e^{-\frac{b}{D}\left(\sqrt{N}-\frac{a}{b}\right)^{2}}}{\operatorname{erfc}\left(-\frac{a}{\sqrt{b D}}\right)},
$$


and the moments of $N$

$$
\mu_{n}=\sqrt{\frac{2}{\pi}} \frac{e^{-\frac{a^{2}}{2 b D}}}{\operatorname{erfc}\left(-\frac{a}{\sqrt{b D}}\right)}\left(\frac{D}{2 b}\right)^{n}(2 n) ! D_{-2 n-1}\left(-\sqrt{\frac{2 a^{2}}{b D}}\right),
$$

where $\operatorname{erfc}(z)$ is the complementary error function and $D_{\nu}(z)$ is the parabolic cylinder function.

\subsection{Spectral density of lemming population}

The idea, presented in Ref. 34, is that the correlation properties of the noise can be taken into consideration by a simple method. The equation is differential and its solution is an integral. Therefore, the moments are defined not by the noise itself but by its integral.

We introduce a new random process $z(t)$ defined as

$$
z(t)=\int_{0}^{t}(\xi(\tau)-\langle\xi\rangle) d \tau
$$

If we consider the process at large times, because of integration procedure, the difference between white and correlated noise is not a qualitative difference but only quantitative, in accordance with the central limit theorem. The variance of this Gaussian distribution is defined by the correlation function of $z(t)$ and can be presented through the correlation function of the noise $\xi(t)$

$$
\sigma_{z}^{2}(t) \approx 2 t \int_{0}^{\infty} K_{\xi-\langle\xi\rangle}(\tau) d \tau=S_{\xi-\langle\xi\rangle}(0) t .
$$

In the case of white noise, $\sigma_{z}^{2}(t)=4 D t$. We propose the hypothesis that the asymptotic dynamics of the moments in the presence of colored noise is the same as it is for white noise with coefficient

$$
D=S_{\xi-\langle\xi\rangle}(0) / 4
$$

Numerical simulations provide support for this hypothesis.

Let us consider a stochastic process $\xi(t)$ consisting of delta pulses with positive magnitudes $f_{i}$

$$
\xi(t)=\sum_{i} f_{i} \delta\left(t-t_{i}\right)
$$

We denote the random distance between adjacent pulses as $\zeta_{i}=t_{i}-t_{i-1}$ and assume that the mean distance is $\langle\zeta\rangle=T$. Also, we assume that $f_{i}$ is uniformly distributed with the variance $\sigma_{f}^{2}$. This means that $\langle\xi(t)\rangle=\langle f\rangle / T$.

The DTDP process ${ }^{35}$ is a renewal pulse process with delay $\zeta_{0}$ after each pulse. During this dead-time period it is forbidden for a new pulse to appear. After this period, the probability per unit time to have the next pulse $p$ is constant. This process is suitable to obtain noise sources with varying degree of randomness. ${ }^{7,32}$ 

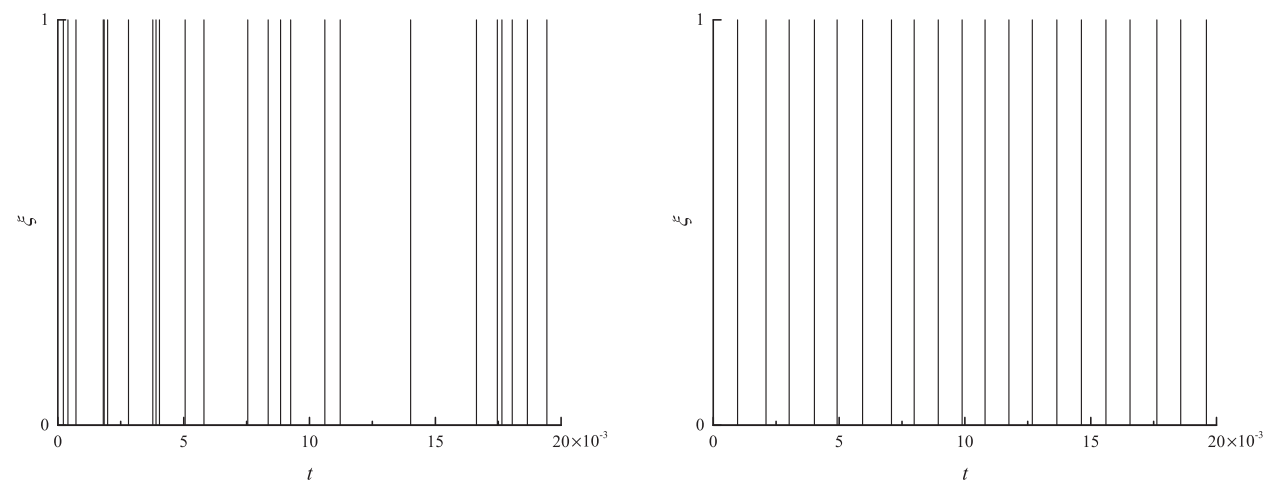

Fig. 2. Realizations of the DTDP process (constant magnitude $f=1$ and $T=0.001$ ). Left panel: totally random process $(\varrho=0)$. Right panel: quasi-periodic process $(\varrho=0.9)$. is $^{32}$

The probability distribution of random time distances $\zeta$ between adjacent pulses

$$
w(\zeta)=H\left(\zeta-\zeta_{0}\right) p e^{-p\left(\zeta-\zeta_{0}\right)},
$$

where $H(x)$ is the Heaviside step function. The mean value and the variance of $\zeta$ are respectively $\langle\zeta\rangle=\zeta_{0}+1 / p=T$ and $\sigma_{\zeta}^{2}=\left(T-\zeta_{0}\right)^{2}$.

In the limit $\zeta_{0} \rightarrow T$ the probability $p \rightarrow \infty$, and we have a deterministic periodical process. In this case the time intervals between pulses are given by the probability distribution $w(\zeta)=\delta\left(\zeta-\zeta_{0}\right)$. Conversely for $\zeta_{0}=0$, the stochastic process $\xi(t)$ coincides with the white Poisson noise. The degree of periodicity, which depends on the memory and average period of the pulse noise, is quantitatively given by $\varrho=\zeta_{0} / T$ and ranges from 0 to 1 . Figure 2 shows two realizations of the DTDP process obtained with different values of the periodicity parameter $\varrho$.

In accordance with Ref. 32, the spectral density for the pulse process under consideration at $\omega=0$ is

$$
S_{\xi-\langle\xi\rangle}(0)=\frac{2\langle f\rangle^{2} \sigma_{\zeta}^{2}}{T^{3}}+\frac{2 \sigma_{f}^{2}}{T} .
$$

\section{Numerical Simulation}

Here, we present results obtained by numerical integration of Eq. (1) where the noise source is the DTDP process of Eq. (10). We perform numerical simulations by using the Mersenne twister ${ }^{36}$ as pseudo-random number generator. The averaging is performed over $10^{6}$ numerical realizations.

In Fig. 3, we show the dynamics of the average predator population density for $\beta=1$, obtained by numerically solving Eq. (1) for two different values of the periodicity parameter, i.e., $\varrho=0$ and 0.9 . We use $b=10$, constant magnitude $f=0.1, T=0.002$, and the delta function initial distribution with $N(0)=5$. In the inset of Fig. 3, we show enlarged fragment of the plot (fluctuating lines) 


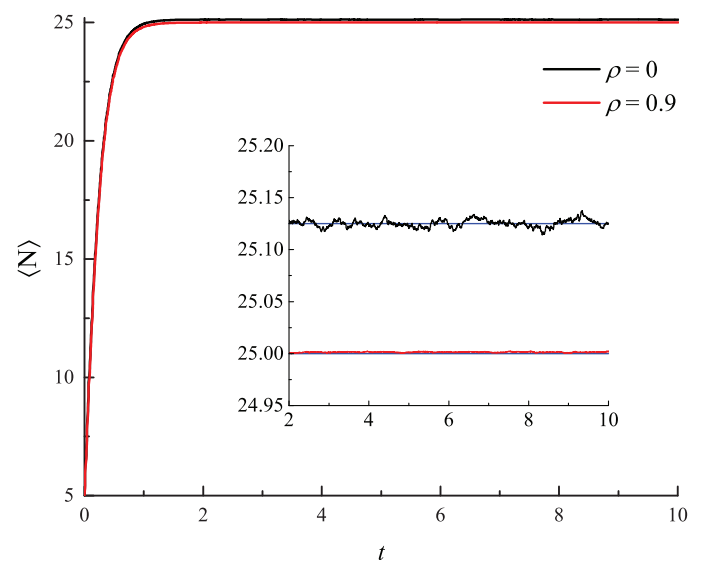

Fig. 3. (Color online) Plot of the average predator population density $\langle N\rangle$ versus time for two different values of $\varrho$. The values of the parameters and the initial condition for $N$ are $\beta=1, b=10$, constant magnitude $f=0.1, T=0.002$ and $N(0)=5$. Red line: Quasi-periodic DTDP noise $(\varrho=0.9)$. Black line: Totally random pulse noise $(\varrho=0)$. Inset: Analytical results derived from Eqs. (6) and (9) (solid horizontal lines), for the same values of periodicity parameter, i.e., $\varrho=0$ and 0.9 , together with the corresponding numerical simulations (fluctuating lines).

together with the corresponding results of analytical calculations using expressions (6) and (9) (solid horizontal lines). We see that the numerical results are in a good agreement with the analytical ones. Specifically for quasi-periodical noise $(\varrho=0.9)$, the numerical results almost coincide with the analytical ones.

Less regular cycles of lemmings cause higher saturation values and large fluctuations in the predator population density, whose dynamics therefore becomes more difficult to predict.

Figure 4 presents the results of simulations for a quadratic dependency of predator's decrease rate $(\beta=2)$, and for the same values of $\varrho$ of Fig. 3. The other parameter values are: $b=1$, constant magnitude $f=0.1, T=0.01$, and Rayleigh initial distribution of $N$ with $\sigma=4$. In the inset of Fig. 4, we show both analytical and numerical results as in Fig. 3.

The case of variable magnitude of the noise pulses is shown in Fig. 5. Here, the parameter values are $\beta=5 / 2, b=0.1, T=0.01$, and the delta function initial distribution corresponds to $N(0)=2$. The magnitude distribution is uniform in the interval $[0,0.2]$. It is easy to see that the numerical solution for the quasi-periodic case $(\varrho=0.9)$ is more "noisy" in comparison with those shown in Figs. 3 and 4 , because $\sigma_{f}^{2} \neq 0$ in Eq. (12). For linear extinction term $(\beta=1)$, a high periodicity in the noise term causes a decrease in the stationary value of the predator population (see Fig. 3). For nonlinear extinction terms $(\beta \geq 2)$, vice versa, a high periodicity in the fluctuations of the prey population causes an increase in the stationary value of the predators (see Figs. 4 and 5). In other words, the increasing nonlinearity of the extinction term of the predator population gives rise to a nonmonotonic dependence of stationary values of the predator population on the periodicity of noise. 


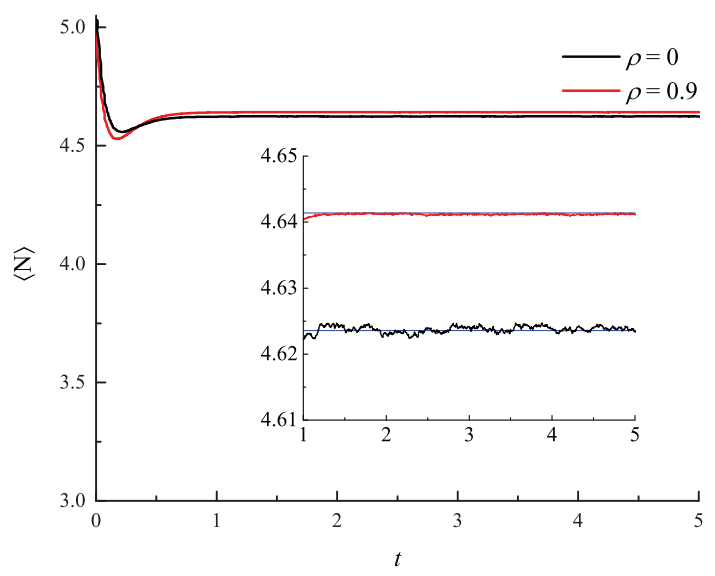

Fig. 4. (Color online) Plot of the average population density $\langle N\rangle$ versus time for two different values of $\varrho$. The values of the parameters are $\beta=2, b=1$, constant magnitude $f=0.1$ and $T=0.01$. The initial distribution of $N$ is Rayleigh distribution with $\sigma=4$. Red line: Quasiperiodic DTDP noise $(\varrho=0.9)$. Black line: Totally random pulse noise $(\varrho=0)$. Inset: Analytical results derived from Eqs. (4) and (9) (solid horizontal lines), for the same values of periodicity parameter $\varrho$, together with the corresponding numerical simulations (fluctuating lines).

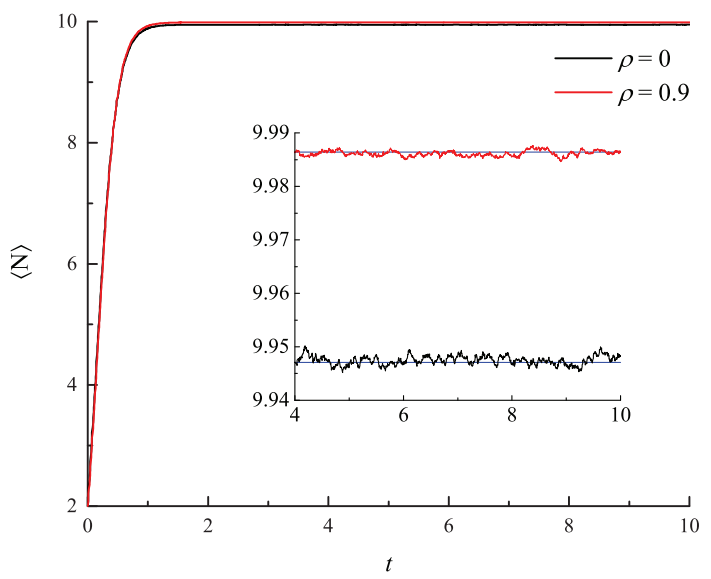

Fig. 5. (Color online) Plot of the average predator population density $\langle N\rangle$ versus time for two different values of $\varrho$. The values of the parameters and the initial condition are $\beta=5 / 2, b=0.1$, uniform distribution of $f$ in $[0,0.2], T=0.01$ and $N(0)=2$. Red line: Quasi-periodic DTDP noise $(\varrho=0.9)$. Black line: Totally random pulse noise $(\varrho=0)$. Inset: Analytical results derived from Eqs. (4) and (9) (solid horizontal lines), for the same values of periodicity parameter $\varrho$, together with the corresponding numerical simulations (fluctuating lines).

In Figure 6 we compare the analytical result of the stationary PDF calculated from Eq. (4), with the PDF calculated at $t=20$, and obtained by numerically solving Eq. (1) for purely random noise $(\varrho=0)$. The parameters are the same as in Fig. 4. The agreement between analytical and numerical PDF is quite good. 


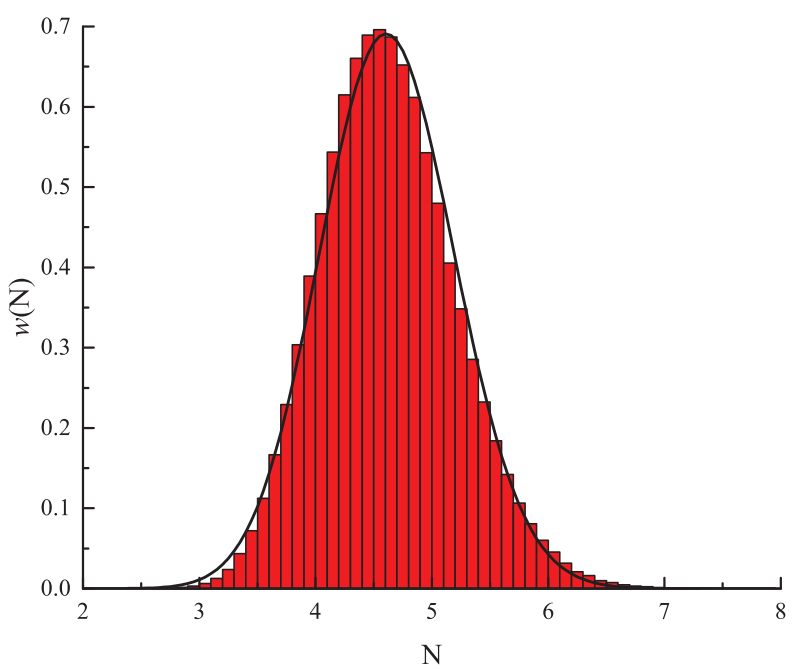

Fig. 6. (Color online) Probability density function of $N$ at $t=20$ for $\varrho=0$, calculated by numerically solving Eq. (1) (histogram). Analytical stationary PDF, calculated from Eq. (4) (solid line). The values of the parameters are $\beta=2, b=1$, constant magnitude $f=0.1, T=0.01$.

\section{Summary}

In this work, we considered an ecosystem consisting of two species, i.e., a predator population and a prey population. This topic is of paramount importance for ecosystems in which a prey population shows the dynamics characterized by large fluctuations. Here we took them into account by inserting a term of multiplicative noise in the equation for the predator dynamics.

The decrease of predators is caused by intraspecific competition, whose effect is represented in our model by a negative term. This term causes the appearance of a stationary state with a saturation value which depends on the noise properties.

We have demonstrated that the contribution to the mean value of population density changes as the periodicity of the noise varies.

For animals in north regions some periodicity or cycling in population density is well-known. Here, we have presented the approach to calculate the influence of the prey cycles modeled as a pulse noise, whose periodicity can be regulated as a function of a delay. In particular, we have showed that a greater periodicity in the prey population gives rise to smaller fluctuations in the predator density. Moreover, the influence of the periodicity depends on the degree of nonlinearity of the extinction term in Eq. (1). If $\beta=1$, the increase of the periodicity causes a decrease in the stationary value of the predator density.

In the case of a stronger dependence $(\beta \geq 2)$ of the intraspecific competition on the population density $N$, the stationary value of predator population itself decreases with the randomness of food availability. More regularity is therefore more advantageous for the predator population. 


\section{References}

1. J. Ripa and P. Lundberg, Proc. R. Soc. Lond. B 263(1377), 1751 (1996).

2. X. Mao, G. Marion and E. Renshaw, Stoch. Process. Appl. 97(1), 95 (2002).

3. D. Valenti, G. Augello and B. Spagnolo, Eur. Phys. J. B 65, 443 (2008).

4. G. Denaro et al., Ecol. Complex. 13, 21 (2013).

5. C. W. Gardiner, Handbook of Stochastic Methods: For Physics, Chemistry and the Natural Sciences (Springer, Berlin, 1985).

6. A. Angerbjorn, M. Tannerfeldt and S. Erlinge, J. Anim. Ecol. 68, 34 (1999).

7. O. A. Chichigina, Eur. Phys. J. B 65(3), 347 (2008).

8. M. Predavec et al., Oecologia 126(2), 216 (2001).

9. G. O. Batzli, Food selection by lemmings, in The Biology of Lemmings, eds N. C. Stenseth and R. A. Ims (Academic Press, London, 1993), pp. 281-301.

10. R. Krebs, R. Boonstra and A. J. Kenney, Oecologia 103, 481 (1995).

11. D. G. Reid, C. J. Krebs and A. Kenney, Oikos 73, 387 (1995).

12. H. Steen, N. G. Yoccoz and R. A. Ims, Oikos 59, 115 (1990).

13. N. C. Stenseth and R. A. Ims, Population dynamics of lemmings: temporal and spatial variation - an introduction, in The Biology of Lemmings, eds. N. C. Stenseth and R. A. Ims (Academic Press, London, 1993), pp. 61-96.

14. A. S. Mikhailov and A. Yu. Loskutov, Foundations of Synergetics II: Chaos and Noise (Springer, Berlin, 1996).

15. M. A. Muñoz, Multiplicative noise in non-equilibrium phase transitions: A tutorial, in Advances in Condensed Matter and Statistical Physics, eds. E. Korutcheva and R. Cuerno (Nova Science Publishers, New York, 2004), pp. 37-68.

16. A. A. Dubkov and B. Spagnolo, Phys. Rev. E 72, 041104 (2005).

17. A. Fiasconaro, D. Valenti and B. Spagnolo, Acta Phys. Pol. B 35, 1491 (2004).

18. M. A. Muñoz, F. Colaiori and C. Castellano, Phys. Rev. E 72, 056102 (2005).

19. A. Manor and N. M. Shnerb, Phys. Rev. Lett. 103, 030601 (2009).

20. G. Bonanno, D. Valenti and B. Spagnolo, Phys. Rev. E 75, 016106 (2007).

21. A. Schenzle and H. Brand, Phys. Rev. A 20, 1628 (1979).

22. R. Graham and A. Schenzle, Phys. Rev. A 25, 1731 (1982).

23. J. García-Ojalvo and J. M. Sancho, Noise in Spatially Extended Systems (Springer, New York, 1999).

24. S. Redner, Am. J. Phys. 58, 267 (1990).

25. D. Sornette, Critical Phenomena in Natural Sciences (Springer, Heidelberg, 2000).

26. S. Ciuchi, F. de Pasquale and B. Spagnolo, Phys. Rev. E 47, 3915 (1993).

27. A. La Cognata et al., Phys. Rev. E 82, 011121 (2010).

28. R. Kree, B. Schaub and B. Schmittmann, Phys. Rev. A 39, 2214 (1989).

29. A. Fiasconaro et al., Eur. Phys. J. B 65, 435 (2008).

30. E. Framstad et al., Proc. R. Soc. Lond. B 264(1378), 31 (1997).

31. D. Valenti et al., Ecol. Model. 213, 449 (2008).

32. O. A. Chichigina et al., Phys. Rev. E 84(2), 021134 (2011).

33. I. S. Gradshteyn and I. M. Ryzhik, Table of Integrals, Series and Products (Elsevier/Academic Press, Amsterdam, 2007).

34. D. Valenti et al., J. Stat. Mech. 2015, P02012 (2015).

35. O. Chichigina, D. Valenti and B. Spagnolo, Fluct. Noise Lett. 5, L243 (2005).

36. M. Matsumoto and T. Nishimura, ACM Trans. Model. Comput. Simul. 8, 3 (1998). 